\title{
Article \\ Relationship between Behavioral Infant Speech Perception and Hearing Age for Children with Hearing Loss
}

\author{
Kristin M. Uhler ${ }^{1, *}$, Alexander M. Kaizer ${ }^{2}$, Kerry A. Walker ${ }^{3}{ }^{(1)}$ and Phillip M. Gilley ${ }^{4}$ \\ 1 Department of Physical Medicine and Rehabilitation, University of Colorado School of Medicine, \\ Children's Hospital Colorado, Aurora, CO 80045, USA \\ 2 Department of Biostatics and Informatics, Colorado School of Public Health, \\ University of Colorado Anschutz Medical Campus, Aurora, CO 80045, USA; alex.kaizer@cuanschutz.edu \\ 3 Department of Otolaryngology-Head \& Neck Surgery, University of Colorado School of Medicine, \\ Aurora, CO 80045, USA; Kerry.walker@cuanschutz.edu \\ 4 Institute of Cognitive Science, University of Colorado, Boulder, CO 80309, USA; gilley@colorado.edu \\ * Correspondence: kristin.uhler@cuanschutz.edu
}

check for updates

Citation: Uhler, K.M.; Kaizer, A.M.; Walker, K.A.; Gilley, P.M. Relationship between Behavioral Infant Speech Perception and Hearing Age for Children with Hearing Loss. J. Clin. Med. 2021, 10, 4566. https://doi.org/ $10.3390 /$ jcm10194566

Academic Editor: Lisa L. Hunter

Received: 1 September 2021

Accepted: 28 September 2021

Published: 30 September 2021

Publisher's Note: MDPI stays neutral with regard to jurisdictional claims in published maps and institutional affiliations.

Copyright: (c) 2021 by the authors. Licensee MDPI, Basel, Switzerland. This article is an open access article distributed under the terms and conditions of the Creative Commons Attribution (CC BY) license (https:// creativecommons.org/licenses/by/ $4.0 /)$.

\begin{abstract}
Background: Research has demonstrated that early intervention for children who are hard-of-hearing $(\mathrm{CHH})$ facilitates improved language development. Early speech perception abilities may impact $\mathrm{CHH}$ outcomes and guide future intervention. The objective of this study was to examine the use of a conditioned head turn (CHT) task as a measure of speech discrimination in $\mathrm{CHH}$ using a clinically feasible protocol. (2) Methods: Speech perception was assessed for a consonant and vowel contrast among $57 \mathrm{CHH}$ and 70 children with normal hearing $(\mathrm{CNH})$ aged 5-17 months using a CHT paradigm. (3) Results: Regardless of hearing status, $74 \%$ of $\mathrm{CHH}$ and $77 \%$ of $\mathrm{CNH}$ could discriminate /a-i/, and $55 \%$ of $\mathrm{CHH}$ and $56 \%$ of $\mathrm{CNH}$ could discriminate /ba-da/. Regression models revealed that both $\mathrm{CHH}$ and $\mathrm{CNH}$ performed better on /ba-da/ at $70 \mathrm{dBA}$ compared to $50 \mathrm{dBA}$. Performance by hearing age showed no speech perception differences for $\mathrm{CNH}$ and children with mild hearing loss for either contrast. However, children with hearing losses $\geq 41 \mathrm{~dB}$ HL performed significantly poorer than $\mathrm{CNH}$ for /a-i/. (4) Conclusions: This study demonstrates the clinical feasibility of assessing early speech perception in infants with hearing loss and replicates previous findings of speech perception abilities among $\mathrm{CHH}$ and $\mathrm{CNH}$.
\end{abstract}

Keywords: infant speech perception; early intervention; congenital hearing loss; hearing aids

\section{Introduction}

This is a study about behavioral speech perception among hard-of-hearing infants whose hearing losses were identified through universal newborn hearing screenings. Infants in this study were fit with hearing aids by 3 months of age and participated in early intervention through a system of care called Early Hearing Detection and Intervention (EHDI) in the United States. Universal newborn hearing screenings and EHDI systems have radically improved age at identification, diagnosis, and early intervention for children who are hard-of-hearing $(\mathrm{CHH})$, allowing speech perception testing at very young ages. While EHDI programs have led to significant improvements in language outcomes, much remains unknown about early speech perception abilities in young infants with hearing loss or how such abilities contribute to language outcomes compared to their normal hearing peers [1]. CHH who benefit from the EHDI programs demonstrate improved language, social-emotional, and academic skills [2-4] compared to children who are diagnosed and treated after two-years of age [2,5-8]. Further, early identification and treatment services have resulted in receptive and expressive language skills similar to children with normal hearing $(\mathrm{CNH})$ [8-10]. Despite these improvements, there continues to be wide variability in spoken language outcomes (i.e., mean vocabulary quotient $=77.6, \mathrm{SD}=19.7$; [11]), word learning abilities [12], and academic achievement [13-15] for $\mathrm{CHH}$. Based on results 
from our previous research, we suggest that such variability in outcomes may be due to differences in speech perception abilities during infancy, which are poorer for infants with hearing loss [16], and may partially be explained by reduced auditory access to the full speech spectrum even after being fitted with hearing aids.

Clinical assessment of speech perception is recommended as part of the Pediatric Minimum Speech Test Battery during the first year of life [17]; however, clinical tools are not readily available to objectively assess and validate hearing aid fittings and appropriateness of intervention strategies (e.g., hearing aid programming, transitioning to cochlear implants, or remote microphone technology). Performing "real-ear measures" is the current best practice for hearing aid verification in measuring the output of hearing aids in the outer ear canal. The sole utilization of hearing aid verification cannot ensure infants have access to the acoustic information needed to discriminate between speech sounds-a foundation for learning spoken language $[6,14,18-21]$. According to a survey of 117 pediatric audiologists, parent questionnaires remain the most frequently used clinical tool to assess speech perception for children under the age of two [22]; however, questionnaires are not objective measures of speech perception. Taken together, the lack of clinical tools and the variability of patient outcomes necessitates the translation of speech perception protocols from a research setting into clinical practice.

Numerous studies among $\mathrm{CHH}$ and $\mathrm{CNH}$ have examined speech perception abilities longitudinally [23-26]. However, we are interested in the first stage of perception as described by Aslin and Smith's 1988 overview of the structural levels of perceptual development [27]. According to their model, perceptual development occurs in hierarchical stages consisting of three elements: (1) the "sensory primitives" or elementary perceptual units, (2) perceptual representations, and (3) higher order representations. Similar to the work of others (e.g., $[25,28]$ ) examining speech perception within this conceptual framework allows us to consider the first stage of development by examining an infant's ability to detect a change in "sensory primitive" units of speech stimuli. A child's inability to discriminate between two speech sounds could have a cascading effect on that child's development of "perceptual representations," and in turn, hinder the development of higher-order representations or the ability to form meaningful words/sentences from simple speech sounds. The inability of children to segment and differentiate speech stimuli from an ongoing stimulus would have deleterious impacts on language outcomes [29-31]. Therefore, it is essential to formulate a fundamental understanding of how infant speech perception develops among $\mathrm{CHH}$ compared to their $\mathrm{CNH}$ peers.

Among $\mathrm{CNH}$, speech perception has been assessed in research settings for over 40 years and can be assessed behaviorally around 6 months of age using a conditioned head turn (CHT) paradigm. CHT is similar to a commonly used audiologic assessment tool, visual reinforcement audiometry (VRA). VRA is used to assess an infant's ability to detect the presence/absence of sound and is clinically useful for establishing an infant's audiometric thresholds [32]. Whereas $\mathrm{CHT}$ has been used to document a CNH's ability to differentiate between vowels (/a-i/) and consonants (/ba-da/), translating this paradigm for clinical use requires systematic manipulation of test time and the number of test sessions while simultaneously maintaining scientific rigor of the test measure. For example, assessing speech perception in $\mathrm{CNH}$ has typically consisted of repeat testing if a child could not reach criterion on the speech perception task (e.g., [33-36]). This demonstrates that infants can improve their performance if they are seen multiple times for perceptual testing in a controlled research setting. CHT protocols have significant variability in attrition rates $(10-15 \% ;[37,38])$ versus past studies which had attrition rates ranging from 5-50\% [39] due to excluding data because infants became fussy, had abnormal middle ear function, or did not meet shaping/training criteria. Commonly, the numbers of children who did not meet shaping/training criteria are aggregated and their scores are not included in the statistical results. This approach to data aggregation leads to challenges in differentiating infants with poor speech discrimination abilities versus those who did not condition to the task or reach training criteria. Furthermore, the utilization of the shaping/training sessions add 
an additional study visit. For example, from $26 \%$ [40] to $33 \%$ of the participants' data [35] has been excluded from previous reports and percentage of excluded participants does not encompass the number of infants who were excluded due to fussiness or ear infections. Taken together, these approaches result in more test sessions and smaller sample sizes. From a clinical perspective, if a child conditions to a task such as VRA, then clinicians may proceed with testing without further training and without detriment to the results. This concept is not novel and is commonly used in other speech perception tests where the child is taught the task such as the Early Speech Perception test [41] and the Open and Closed Set Speech Perception Test [42] using two to three practice words prior to test initiation. In the present study, we took a similar conditioning approach to Tsao and colleagues [30] here we conditioned to the task and then initiated testing once a child demonstrated task competency which is essential for future clinical utility.

Assessment of speech perception among $\mathrm{CNH}$ has demonstrated that infants transition from being universal language learners and become better at discriminating their native languages between 6-12 months of age [43-46] as shaped by their language environment. Among $\mathrm{CNH}$, speech perception abilities change over the first year of life as they become better at discriminating sounds in their native language. Infants also require a greater (louder) presentation level than adults to discriminate speech sounds [34-36,47] and the speech sound of interest must be louder than the background noise for infants to successfully differentiate between / ba/ and / da/. Nozza [36] assessed multiple intensity levels $(50,60$, and $70 \mathrm{dBA})$ and compared performance for each intensity level to the softest sound both infants and adults could detect. For successful discrimination between the $/ \mathrm{ba} /$ and the $/ \mathrm{da} /$ the presentation level had to be 10-15 dB greater for infants compared to adults. These results suggest that utilizing a single intensity level as used for adults may result in poorer speech perception scores among infants [26,40,48,49].

While there is still more to learn about the development of speech perception among infants with normal hearing, even less is known about how development is impacted by the presence of hearing loss. Much of the work on speech perception development has been done in infants who use cochlear implants. Little is known about speech perception among infants with mild to severe permanent hearing loss between 6 to 17 months of age who benefited from early identification and hearing aid use. One finding is that $\mathrm{CHH}$ in the mild to severe hearing loss range discriminate vowel sounds more accurately than consonant sounds $[26,37,38,48,50,51]$. In our previous work, $\mathrm{CHH}$ (while wearing HAs) and $\mathrm{CNH}$ performed similarly on vowel discrimination (/a/ versus / $\mathrm{i} /$ ). In contrast, only $50 \%$ of $\mathrm{CHH}$ and $71 \%$ of $\mathrm{CNH}$ discriminated $/ \mathrm{b} /$ and $/ \mathrm{d} /$ (Uhler et al. [37]). Among $\mathrm{CHH}$ only, vowel discrimination was better compared to consonant discrimination $(p=0.004)$, but the same was not true for the $\mathrm{CNH}(p=0.45)$.

The majority of speech perception studies for $\mathrm{CHH}$ have involved young deaf children who receive cochlear implants between 6-24 months of age and assessed speech perception between 9-30 months of age using CHT measures [26,52,53] visual habituation [24] or a modified visual habituation task [25]. Different speech stimuli have been employed, such as vowel stimuli $[25,26,52]$, consonant contrasts [26,52], and suprasegmental differences (ahhh vs. hop hop) [54]. Broadly these findings suggest that following cochlear implantation, speech perception improves over time for young cochlear implant users. In contrast, Horn, Houston, and Miyamoto [23] found that following 1.4 months of cochlear implant use, 17-month-old listeners, were unable to discriminate "seepug" versus "boodup" through audition alone. When compared to their normal hearing peers, $\mathrm{CHH}$ may have different auditory development trajectories depending on various factors such as age at fit or degree of hearing loss.

In this study, we extend our work towards a tool that could be clinically useful to assess speech discrimination in infancy $[37,39]$. A tool to objectively assess speech perception during infancy could help to determine if $\mathrm{CHH}$ are fitted appropriately and if they have access to acoustic cues allowing for speech discrimination (i.e., validation 
of hearing aid fittings). We set out to address three primary questions using a clinically feasible testing schedule:

1. What proportion of $\mathrm{CHH}$ and $\mathrm{CNH}$ can demonstrate discrimination of each a vowel and consonant contrast as measured by CHT, at 50, 60, and/or $70 \mathrm{dBA}$ SPL in a single test session while accounting for hearing age?

2. Is there a difference in the ability of $\mathrm{CHH}$ (who met 1-3-6 benchmarks) and $\mathrm{CNH}$ in their demonstration of vowel and consonant discrimination assessed through a CHT task?

3. Is there a relationship between aided SII measured at $50 \mathrm{~dB}$ SPL and performance on a CHT speech perception task for each contrast?

\section{Materials and Methods}

\subsection{Participants}

Data were collected for 129 children $(58 \mathrm{CHH}$ and $71 \mathrm{CNH})$ aged $5-17$ months (mean ( $\mathrm{M}=9.61$ months, standard deviation (SD) $=2.34$ months. Two children (one $\mathrm{CNH}$ and one $\mathrm{CHH}$ ) were lost to follow up prior to collecting speech perception data resulting in 127 children contributing to speech perception data $(57 \mathrm{CHH}, 70 \mathrm{CNH}) .110$ children completed speech perception testing for both consonant and vowel contrasts (/a/vs. /i/ and /ba/ vs. /da/) the remaining 17 children completed either /a-i/ or /ba-da/. Specifically, nine CNH and two $\mathrm{CHH}$ only completed /a-i/ and three $\mathrm{CNH}$ and three $\mathrm{CHH}$ only completed the /ba-da/ contrast. The reasons for data loss were as follows: failure to condition to the task for the second contrast (three $\mathrm{CNH}$, three $\mathrm{CHH}$ ), loss to follow-up after completing one contrast (one $\mathrm{CNH}$, one $\mathrm{CHH}$ ), and modification in the presentation of the stimuli level for /ba-da/ 50 versus $65 \mathrm{dBA}$ SPL for the first level assessed (eight $\mathrm{CNH}$, one $\mathrm{CHH}$ ). The data for 43 children with $\mathrm{CNH}$ [16] and 11 children with $\mathrm{CHH}$ [16] have also been reported in previous studies. Amplification and speech perception data were obtained from 57 infants (28 males and 29 females) with bilateral sensorineural hearing losses ranging from mild to severe. The better-ear pure tone average $(500,1000,2000$, and $4000 \mathrm{~Hz})$ for the $\mathrm{CHH}$ group ranged from 18.33 to $83.33 \mathrm{~dB} \mathrm{HL}(\mathrm{M}=41.3 \mathrm{~dB}, \mathrm{SD}=14.6 \mathrm{~dB})$. All $\mathrm{CHH}$ wore bilateral air conduction hearing aids. The age at hearing aid fitting ranged from 1 to 6 months ( $\mathrm{M}=2.92$ months; $\mathrm{SD}=1.24$ months), except for two children who were fit at 8 months of age. These two children were enrolled in early intervention, but their parents chose to delay fitting of hearing aids. For comparison purposes, data were obtained on $70 \mathrm{CNH}$ (38 males and 32 females). The demographics for $\mathrm{CHH}$ and $\mathrm{CNH}$ appears in Table 1.

Table 1. Participant Characteristics with results presented as mean (standard deviation) for continuous measures and frequency (percent) for categorical measures.

\begin{tabular}{ccccc}
\hline Demographic & CHH & CNH & Statistical Test & $p$-Value \\
\cline { 2 - 5 } & $\mathbf{( N = 5 7 )}$ & $\mathbf{( N = 7 0 )}$ & & \\
Male & $28(49.1 \%)$ & $38(54.3 \%)$ & Chi-squared & 0.69 \\
Age 9 months or Greater & $30(52.6 \%)$ & $28(40.0 \%)$ & Chi-squared & 0.21 \\
Hearing Age 9 months or Greater & $14(24.6 \%)$ & $28(40.0 \%)$ & Chi-squared & 0.1 \\
Age Category: & & & Fisher's exact & 0.006 \\
6 to 7 months & $7(12.3 \%)$ & $19(27.1 \%)$ & \\
8 to 10 months & $32(56.1 \%)$ & $42(60.0 \%)$ & \\
11 to 13 months & $13(22.8 \%)$ & $3(4.3 \%)$ & \\
14 to 17 months & $5(8.8 \%)$ & $5(7.1 \%)$ & \\
Age $<$ 5 months & $0(0.0 \%)$ & $1(1.4 \%)$ & \\
PTA Category: & & & \\
Normal & $0(0.0 \%)$ & $70(100.0 \%)$ & \\
(0-15 dB HL) & & & \\
\hline
\end{tabular}


Table 1. Cont.

\begin{tabular}{|c|c|c|c|c|}
\hline \multirow{2}{*}{ Demographic } & $\mathrm{CHH}$ & $\mathrm{CNH}$ & Statistical Test & $p$-Value \\
\hline & $(N=57)$ & $(N=70)$ & & \\
\hline $\begin{array}{c}\text { Mild } \\
(16-40 \mathrm{~dB} \text { HL) }\end{array}$ & $39(68.4 \%)$ & $0(0.0 \%)$ & & \\
\hline $\begin{array}{l}\text { Moderate+ } \\
(\geq 1 \mathrm{~dB} \text { HL })\end{array}$ & $18(31.5 \%)$ & $0(0.0 \%)$ & & \\
\hline Hearing Age Category: & & & Fisher's exact & $<0.001$ \\
\hline 0 to 5 months & $19(33.3 \%)$ & $1(1.4 \%)$ & & \\
\hline 6 to 7 months & $22(38.6 \%)$ & $19(27.1 \%)$ & & \\
\hline 8 to 10 months & $11(19.3 \%)$ & $42(60.0 \%)$ & & \\
\hline 11 to 13 months & $5(8.8 \%)$ & $3(4.3 \%)$ & & \\
\hline 14 to 17 months & $0(0.0 \%)$ & $5(7.1 \%)$ & & \\
\hline PTA: & $41.3(14.6)$ & $15.0(0.0)$ & & \\
\hline Missing & $2(3.51 \%)$ & $0(0.0 \%)$ & & \\
\hline Age at CHT in months (M, SD): & $10.2(2.34)$ & $9.16(2.26)$ & $t$-test & 0.02 \\
\hline Hearing Age in months (M, SD): & $7.05(2.49)$ & $9.16(2.26)$ & $t$-test & $<0.001$ \\
\hline Missing & $0(0.0 \%)$ & $70(100.0 \%)$ & & \\
\hline
\end{tabular}

Note. This table summarizes participant characteristics for $\mathrm{CNH}$ and $\mathrm{CHH}$ including gender, mean chronological age at testing, mean hearing age at testing (Chronological age minus age at hearing aid fitting), threshold for /a/, and unaided pure tone audiometry (PTA), all children with hearing loss had bilateral permanent hearing loss.

Inclusion criteria were the same as in Uhler [41] (a) no evidence of significant developmental delays or secondary disabilities per parent report or as indicated in the electronic medical record, (b) demonstrated conditioned head turn in VRA, (c) normal tympanometry on the day of testing or patent pressure equalization tubes, (d) enrollment in early intervention, (e) use of hearing aids per parent report, and (f) either English or Spanish as the primary language spoken in the home. Additional inclusion criteria for the $\mathrm{CNH}$ were $(\mathrm{g})$ parent report of infants passing their newborn hearing screening and the presence of otoacoustic emissions (OAEs) in both ears. Criteria for exclusion were (a) a history of untreated chronic middle ear infections paired with abnormal tympanometric findings on the day of testing and (b) auditory neuropathy.

\subsection{Participant Hearing Aids}

All participants used their own hearing aids during the CHT procedure. Following diagnosis, children received individualized care from their managing audiologist following best practices for amplification fitting, verification, and validation [55]. Children were fit with bilateral, behind-the-ear, air-conduction hearing aids coupled to custom earmolds with appropriate tubing, and filtered ear hooks. Hearing aids were programmed to prevent unnecessary signal distortion and managing audiologists confirmed that all participants' hearing aids were programmed using Desired Sensation Level v5.0 (DSL) [56]. To verify amplification, simulated real-ear response measurements were compared to age-specific DSL targets using real-ear to coupler differences (RECDs). When RECDs were unable to be recorded from at least one ear from the child, average age specific RECD values were substituted. Before laboratory testing, all devices were evaluated to assure proper function. An electroacoustic test box measure was completed to assess hearing aid function. To assess hearing aid output either, measured or simulated real ear coupler differences. The aided SII was automatically calculated at 50, 60, and 70 dBA SPL using the Audioscan Verifit.

Hearing aid use was determined by reading the average daily hearing aid use from the child's hearing aid. The mean hearing aid use was $6.62 \mathrm{~h}(\mathrm{SD}=4.08)$, see Table 2 for demographic characteristics unique to $\mathrm{CHH}$. Each $\mathrm{CHH}$ had speech awareness thresholds and unaided pure-tones assessed using VRA. For the $\mathrm{CNH}$, hearing was screened either using the same procedures as the $\mathrm{CHH}$ or utilization of otoacoustic immittance measures.

Parents were paid \$15 per hour for their child's participation for each study visit. 
Table 2. Demographics unique to $\mathrm{CHH}$.

\begin{tabular}{|c|c|c|}
\hline & Mean (SD/\%) & Median (Min, Max) \\
\hline Age at Hearing Aid Fit & 2.92 months (1.24) & 2.69 months $(1.12,8.03)$ \\
\hline Datalogging & $6.52 \mathrm{~h}(4.12)$ & $6.55 \mathrm{~h}(0.00,16.00)$ \\
\hline Missing & \multicolumn{2}{|c|}{$9(15.8 \%)$} \\
\hline Aided SII: & \multirow{3}{*}{$0.73(0.18)$} & \multirow{3}{*}{$0.74(0.21,0.98)$} \\
\hline $50 \mathrm{dBA}$ & & \\
\hline Missing & & \\
\hline
\end{tabular}

Note. Demographic variables for $\mathrm{CHH}$, including age at hearing aid fitting, datalogging (average use per day), and aided SII for $50 \mathrm{~dB}$ input.

\subsection{Stimuli}

For this experiment, two speech sound contrasts were used, /a-i/ and /ba-da/. Contrasts were selected based on difficulty levels, with vowels being the easiest and consonant contrasts being more difficult for both $\mathrm{CHH}$ and $\mathrm{CNH}[38,50,51,57]$. Our natural speech tokens were produced by a female speaker, and adult listeners in the laboratory verified that the stimuli sounded natural. For procedures on how the stimuli were created, please refer to Uhler et al. [26]. All speech tokens were $500 \mathrm{~ms}$ in duration. For the /ba/ and /da/ stimuli, each consonant was $100 \mathrm{msec}$ in duration, and the vowel duration was $400 \mathrm{msec}$. Stimuli were presented with a $1200 \mathrm{msec}$ interstimulus interval during testing. All stimuli were equated for intensity via root-mean-square (RMS) amplitude normalization. Stimuli were presented at either 50,60, and/or $70 \mathrm{dBA}$ SPL. Sound pressure level of stimuli were measured in the sound field using an A-weighted scale and will be further referred to as dBA. Figure 1 shows a spectrogram of each stimulus to visualize formant differences.

\subsection{Testing Protocol}

All testing was conducted in an acoustically treated sound booth over two sessions. Session one included the case history (information related to the infant's general health, development, and years of education of the infant's mother), tympanometry, hearing screening, and, if time allowed, a threshold search for /a/ using CHT. Session two included a threshold search for /a/ (if not completed at the first visit) and the CHT assessment protocol.

During CHT testing, one of the speech sounds for each stimulus pair, /a/ or / i/ and /ba/ or /da/, served as the background stimulus, repeated with $1200 \mathrm{~ms}$ interstimulus interval throughout the session. The other speech sound served as the target. The member of the pair serving as the target stimulus was counter-balanced across participants. The infant learned to respond by turning their head when the target stimulus was presented.

The infant was accompanied by their caretaker into the sound booth for the CHT assessment. The infant was seated either on the caretaker's lap or in a highchair in the center of the room to minimize distractions or task fatigue. Care was taken to ensure that the infant was comfortable and the location and distance to the speaker remained constant regardless of how the infant was seated. The background stimulus was on when the infant and caretaker entered the room. The speaker and visual reinforcement video screen were $90^{\circ}$ to the right of the infant's midline. An assistant who centered the infant's gaze was positioned in front of the infant, slightly to the left. The caretaker and the assistant listened to music through supra-aural headphones to prevent them from hearing the sounds presented to the infant and inadvertently reinforcing the child or alerting the child to a contrast stimulus.

The evaluator was seated outside the sound booth in a test room and observed the infant through a window. The evaluator in this study was one of five audiologists. The perception task consisted of two phases: conditioning and testing. During the conditioning phase, only change trials were presented so infants could learn to associate a change in the sound and the reinforcer. To facilitate learning during conditioning, the target sound was presented at $6 \mathrm{dBA}$ louder than the background sound to draw the infant's attention to the 
sound change. Initially, in conditioning trials, the reinforcer turned on following two target sounds to "teach" the task to the infant. The infant learned to pair a change in the sound with the reinforcer. After the infant made two consecutive head turns that occurred before the end of the first two presentations of the target sounds the intensity cue was removed. Once testing began, the evaluator could not hear the stimuli and was blinded to stimulus type-trials were initiated by the evaluator by pressing a button when the child's attention was directed toward the midline.
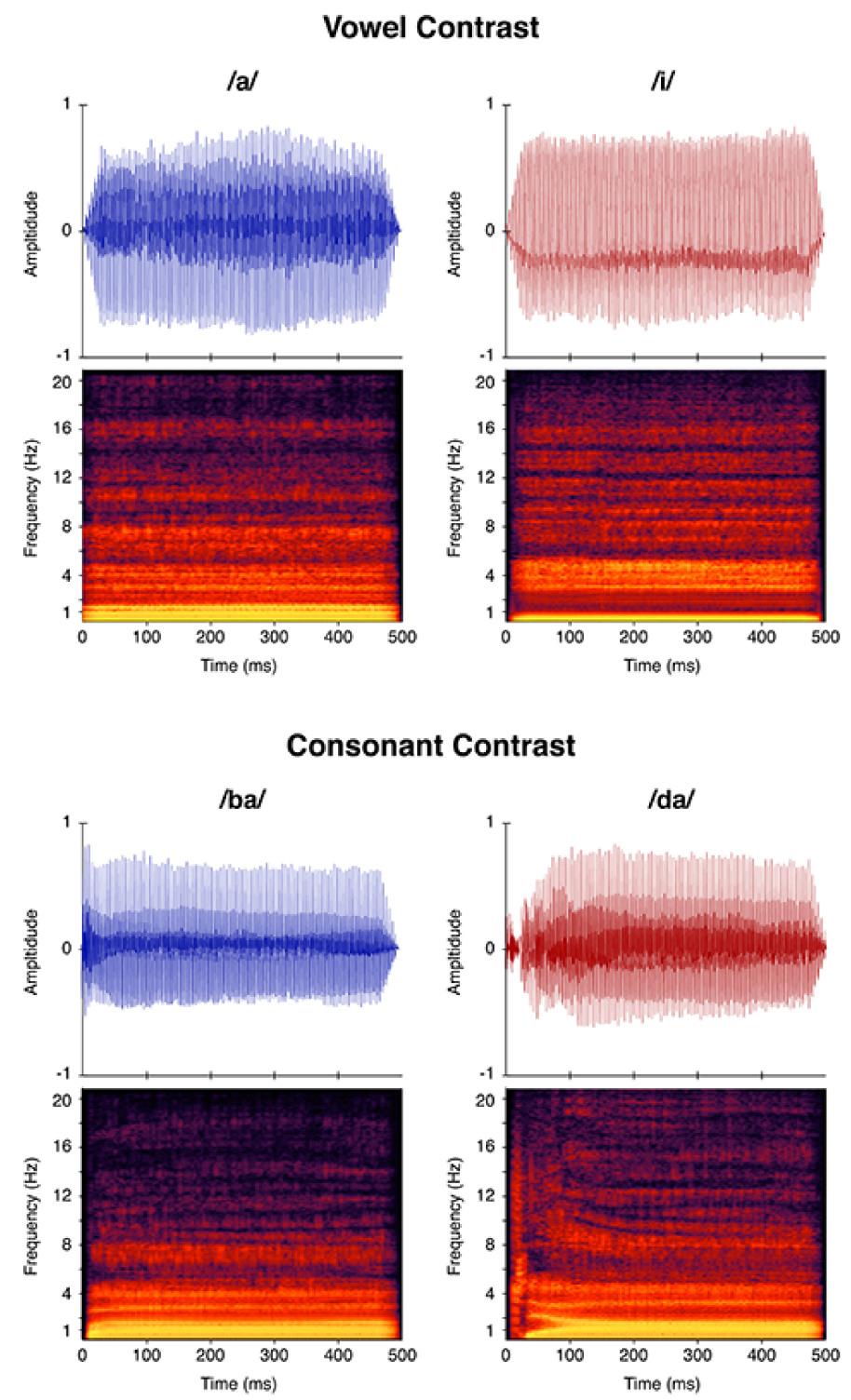

Figure 1. Time-amplitude waveforms and spectrograms for stimuli tested during the speech perception task. The top panel shows the /a/ and /i/ speech sounds for the vowel contrast and the bottom panel shows the /ba/ and / da/ speech sounds for the consonant.

Each of the 15 trials had an equal probability of being a change or no change trial; the trial type was randomly selected by the computer program. If the trial was a no-change trial, the background sound was presented three times. When a change trial was presented, the target sound was presented three times. Once the trial ended, the background sound continued. When head turns were observed by the evaluator, a button was pressed indicating the response. Correct responses were determined by the CHT software, and were rewarded by automatic presentation of an animated video. If the child's head turn was incorrect, it was considered a false positive because no change had occurred. 
Fifteen trials were administered during each contrast assessment. Performance on speech perception was calculated using d-prime $\left(d^{\prime}\right)$ [58]. The advantage of $d^{\prime}$ is that it eliminates the effect of response bias calculated from the number of false positives and hit rate. A "false positive" occurs when a child turned their head, but no change occurred in the stimuli (e.g., a-a-a). In this case, the button indicating that a head turn occurred would be pressed, but no reinforcement would occur. In contrast, a "hit" is when a child turns their head in response to a change in stimuli, which in turn would lead to the behavior being rewarded. False alarm rate is calculated by dividing the number of false alarms by total number of no change trials, and the hit rate is calculated by dividing the number of hits by the total number of change trials. $\mathrm{D}^{\prime}$ is calculated by using the z-score:

$$
\mathrm{d}^{\prime}=\mathrm{z} \text { (false alarms) }-\mathrm{z} \text { (hits) }
$$

If the child achieved a d' value of at least 1.21 at $50 \mathrm{dBA}$, then testing was complete [34,36,38]. We hypothesized that if a child could successfully discriminate at a lower intensity level they would be able to do so at a higher intensity level [59]. For children who did not reach criterion at $50 \mathrm{dBA}$, the level was increased to $70 \mathrm{dBA}$, and testing resumed. Following completion of 15 trials at $70 \mathrm{dBA}$, regardless of performance, the presentation level was reduced to $60 \mathrm{dBA}$, and 15 trials were completed at that presentation level. Therefore, children who did not reach criterion at $50 \mathrm{dBA}$, a total of three conditions (/a-i/ at 50, 60, and $70 \mathrm{dBA}$ ) were completed, see Figure 2. In each session, testing continued until all conditions were completed or if the child was too fussy or tired to continue. On average, a single condition (i.e., /a-i/ at $50 \mathrm{dBA}$ ) was completed in five minutes and $32 \mathrm{~s}(\mathrm{SD}=5.35 \mathrm{~min})$.

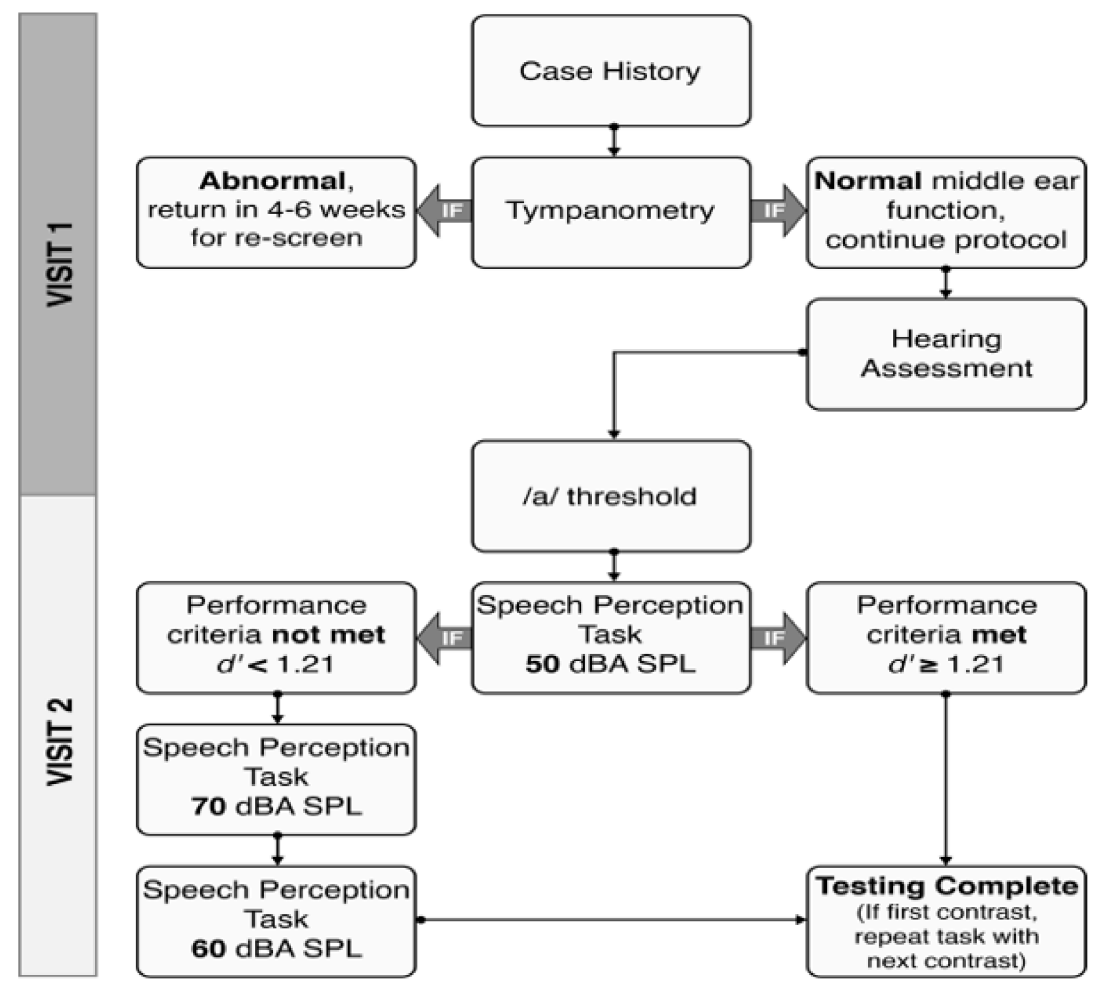

Figure 2. Flow-chart representing the testing protocol consisting of two visits: visit 1 is shown in the upper half of the chart and visit 2 in the lower half of the chart.

\subsection{Statistical Analysis}

Hearing age was calculated by subtracting age at hearing aid fitting (average age at hearing aid fitting was 3 months) from chronological age at test time. $\mathrm{CNH}$ hearing age was the same as chronological test age. Age was stratified as younger or older than nine months at time of testing, chosen as it matches the hearing age of $\mathrm{CHH}$ with the earliest age category 
of $\mathrm{CNH}$ that completed $\mathrm{CHT}$ testing (6-7 months) and better reflects an equal amount of auditory access between groups. Hearing status was categorized as normal hearing, mild hearing loss, and moderate or greater (moderate+) hearing loss. Hearing status and hearing age were analyzed as categorical variables instead of continuous variables given our intent to replicate previous literature with interpretable findings $[11,60]$.

Descriptive summaries are provided as mean (standard deviation, SD) for continuous measures and as frequency (\%) for categorical measures. Normality of the outcomes was evaluated with the Shapiro-Wilk test and graphically, with results suggesting normality. Comparisons of between group demographics used $t$-tests for continuous measures and chi-squared or Fisher's exact tests for categorical measures, as appropriate.

Linear regression models for the outcome of speech perception performance reported as d' scores [58] were evaluated using generalized estimating equations with an exchangeable working correlation structure to account for individuals with multiple scores across different levels of intensity. Models adjusted for hearing age at testing, hearing status, and presentation level as predictors of speech perception performance. Among $\mathrm{CHH}$, models were also fit to include aided SII at $50 \mathrm{dBA}$ as a predictor of speech perception performance.

Scatterplots with speech perception scores across presentation level and hearing status were created with regression fits based on models including PTA category (i.e., normal hearing $0-15 \mathrm{~dB}$ HL, mild $16-40 \mathrm{~dB} \mathrm{HL}$, and moderate $+\geq 41 \mathrm{~dB}$ HL) and presentation level, with points jittered within a given intensity level $(50,60,70 \mathrm{~dB})$ to better visualize the data. All analyses and figures were conducted using R Foundation for Statistical Computing v3.6.3 (Vienna, Austria).

\section{Results}

\subsection{Effect of Hearing Status on Speech Perception}

Table 3 lists the proportion of infants who reached criterion $\left(\mathrm{d}^{\prime} \geq 1.21\right)$ on each contrast as a function of presentation level and hearing status. Participants were included for the lowest presentation level at which they reached criterion and participants that did not reach criterion at any presentation level were placed in the "did not qualify" category for the respective contrast. Percentage totals were created using the total number of participants that completed testing for each respective contrast. For the vowel contrast, among $\mathrm{CHH}, 37 \%$ reached criterion on /a-i/ at $50 \mathrm{dBA}$ and $74 \%$ reached criterion on /a-i/ at any presentation level. Similarly, among $\mathrm{CNH}, 31 \%$ reached criterion at $50 \mathrm{dBA}$ and $77 \%$ reached criterion on /a-i/ at any presentation level. For the consonant contrast, both $\mathrm{CHH}$ and $\mathrm{CNH}$ groups performed similarly with $55 \%$ and $56 \%$ of each group, respectively reaching criterion at any presentation level. Therefore, most infants were able to discriminate between /a-i/ regardless of hearing status and only slightly more than half of the cohort could discriminate /ba-da/. Specifically, among $\mathrm{CHH}$ and $\mathrm{CNH}$ fewer children successfully discriminated /ba-da/ compared to /a-i/.

\subsection{Differences in Speech Perception Abilities among $\mathrm{CHH}$ and $\mathrm{CNH}$}

For analysis, we compared the average speech perception performance for both /a-i/ and /ba-da/ contrasts stratified by hearing age group ( $<9$ months vs. $>9$ months), presentation level, and hearing status. There were no differences between $\mathrm{CHH}$ and $\mathrm{CNH}$ across conditions ( $t$-test $p>0.05)$.

\subsection{Hearing Status}

Next, we categorized speech perception performance as a function of hearing status (normal hearing, mild hearing loss, and moderate+ hearing loss) while considering hearing age. Performance on /a-i/ as a function of presentation level is shown in Figure 3a, and performance on /ba-da/ as a function of presentation level is shown in Figure 3b. Among $\mathrm{CNH}$ and children with mild hearing loss, the regression lines nearly overlap for performance on both contrasts. Children with moderate+ hearing losses demonstrated poorer performance for both the vowel and consonant contrasts than children with lesser degrees of hearing loss. 
For the /a-i/ contrast, there is a significant difference $(\mathrm{W}=-2.14, p=0.03)$ such that children with moderate+ hearing losses had d' scores that were 0.45 lower $(95 \%$ CI $(-0.86,-0.04))$ than CNH. There were no group differences for /ba-da/ ( $p>0.05$ for all).

Table 3. Summary of speech perception.

\begin{tabular}{|c|c|c|c|c|c|c|c|}
\hline \multirow[b]{2}{*}{ Contrast } & \multirow[b]{2}{*}{$\begin{array}{l}\text { Level dBA } \\
\text { SPL }\end{array}$} & \multicolumn{2}{|c|}{$\mathrm{CHH}$} & \multicolumn{4}{|c|}{$\mathrm{CNH}$} \\
\hline & & $\begin{array}{l}\text { Number of } \\
\text { Participants }\end{array}$ & $\begin{array}{c}\% \text { of } \\
\text { Participants }\end{array}$ & $\begin{array}{c}\text { Mean } \\
\text { Performance } \\
\text { in d' (S.D.) }\end{array}$ & $\begin{array}{l}\text { Number of } \\
\text { Participants }\end{array}$ & $\begin{array}{c}\% \text { of } \\
\text { Participants }\end{array}$ & $\begin{array}{c}\text { Mean } \\
\text { Performance in } \\
\text { d' }^{\prime} \text { (S.D.) }\end{array}$ \\
\hline \multirow{3}{*}{ /a-i/ } & 50 & 20 & 37 & $1.94(0.41)$ & 21 & 31 & $1.87(0.46)$ \\
\hline & 60 & 9 & 17 & $1.94(0.46)$ & 25 & 37 & $2.02(0.54)$ \\
\hline & 70 & 11 & 20 & $1.66(0.34)$ & 6 & 9 & $1.80(0.32)$ \\
\hline Did not qualify & & 10 & 19 & & 11 & 16 & \\
\hline $\begin{array}{l}\text { Did not qualify, } \\
\text { some missing }\end{array}$ & & 4 & 7 & & 4 & 6 & \\
\hline Completed Testing & & 54 & & & 67 & & \\
\hline Did not test & & 3 & & & 3 & & \\
\hline \multirow{3}{*}{ /ba-da/ } & 50 & 9 & 16 & $1.88(0.61)$ & 13 & 21 & $1.90(0.62)$ \\
\hline & 60 & 10 & 18 & $1.77(0.60)$ & 8 & 13 & $1.98(0.58)$ \\
\hline & 70 & 12 & 22 & $1.65(0.31)$ & 13 & 21 & $1.97(0.63)$ \\
\hline Did not qualify & & 20 & 36 & & 25 & 41 & \\
\hline $\begin{array}{l}\text { Did not qualify, } \\
\text { incomplete testing }\end{array}$ & & 4 & 7 & & 2 & 3 & \\
\hline Completed Testing & & 55 & & & 61 & & \\
\hline Did not test & & 2 & & & 9 & & \\
\hline
\end{tabular}

Note: Data were included for the lowest level at which criterion was reached for the respective contrast. Due to rounding percentages to the nearest whole number, totals do not add up to $100 \%$.

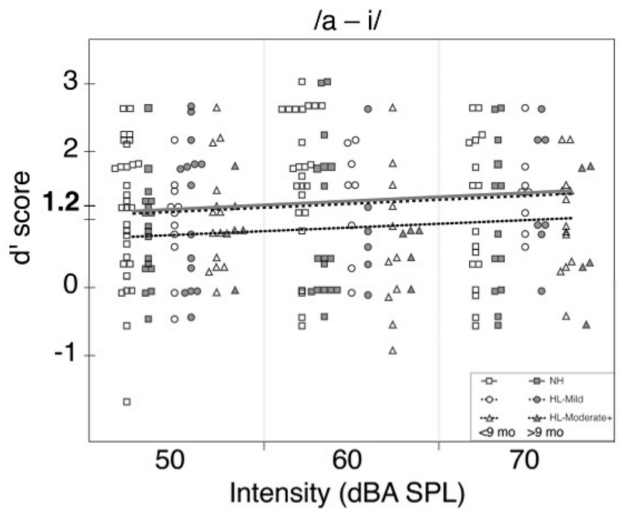

(a)

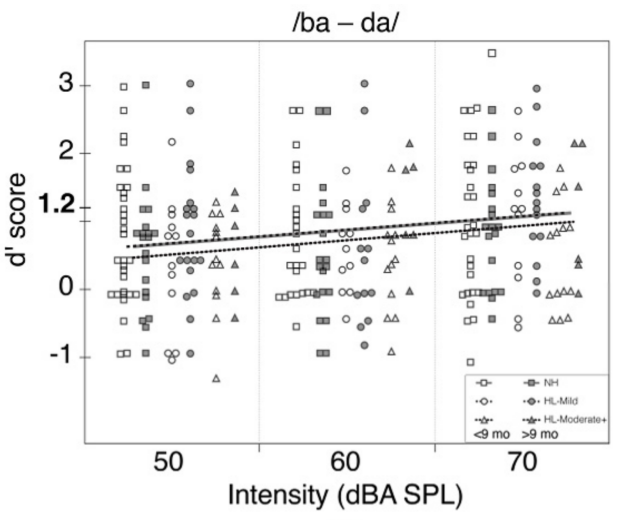

(b)

Figure 3. (a) Performance on /a-i/ as a function of intensity (50, 60, and $70 \mathrm{dBA}$ SPL) represented in columnar format. Each intensity column contains d' scores for each of three hearing groups: normal hearing ( $\mathrm{NH}$; squares), mild hearing loss (HL-mild; circles), and moderate+ hearing loss (HL-moderate+; triangles). Empty symbols represent participants with an age at test less than nine months and filled symbols represent participants with an age at test greater than nine months. Regression lines for each of the hearing groups as a function of intensity level are plotted across the intensity columns (thick grey line $=$ NL; dashed line $=$ HL-mild; dotted line $=$ HL-moderate + ). (b) Performance on $/$ ba-da $/$ as a function of intensity (50, 60, and $70 \mathrm{dBA}$ SPL) represented in columnar format. Each intensity column contains d' scores for each of three hearing groups: normal hearing ( $\mathrm{NH}$; squares), mild hearing loss (HL-mild; circles), and moderate+ hearing loss (HL-moderate+; triangles). Empty symbols represent participants with an age at test less than nine months and filled symbols represent participants with an age at test greater than nine months. Regression lines for each of the hearing groups as a function of intensity level are plotted across the intensity columns (thick grey line = NL; dashed line = HL-mild; dotted line $=$ HL-moderate + ). 


\subsection{Presentation Level}

To examine the role of presentation level for vowel and consonant speech perception abilities, we fit linear regression models for the overall sample of $\mathrm{CHH}$ and $\mathrm{CNH}$ for the outcome of speech perception performance across all presentation levels (50, 60, and $70 \mathrm{dBA}$ ). For the overall sample in Table 4, both $\mathrm{CHH}$ and CNH performed better on /ba-da/ at $70 \mathrm{dBA}$ compared to $50 \mathrm{~dB}$ presentation level and had d' score that was 0.36 higher $(95 \% \mathrm{CI}$ $(0.13,0.58))$ when adjusting for hearing age and hearing status $(\mathrm{W}=3.09, p=0.002)$. Among $\mathrm{CHH}$ cohort, Table 5 , there is improvement in performance as a function of presentation level for / ba-da/ such that at $70 \mathrm{dBA}$ compared to $50 \mathrm{dBA}$ performance was 0.47 higher $(95 \%$ CI $(0.17,0.77))$ adjusting for hearing age and severity of hearing loss. No variables were significantly associated for /a-i/ for the cohort of $\mathrm{CHH}$.

Table 4. Linear regression models for $\mathrm{CHH}$ and $\mathrm{CNH}$.

\begin{tabular}{|c|c|c|c|c|}
\hline Covariate & Estimate & $95 \%$ CI & Wald Statistic (W) & $p$ Value \\
\hline \multicolumn{5}{|c|}{ Model 1-Outcome: d' /ba-da/ for Overall Sample } \\
\hline Intercept & 0.28 & $(-0.32,0.88)$ & 0.90 & 0.37 \\
\hline Hearing Age at Test & 0.04 & $(-0.02,0.1)$ & 1.33 & 0.18 \\
\hline Mild HL (vs. Normal) & 0.07 & $(-0.24,0.38)$ & 0.46 & 0.65 \\
\hline Mod+ HL (vs. Normal) & -0.02 & $(-0.42,0.38)$ & -0.08 & 0.94 \\
\hline 60 Intensity (vs. 50 Intensity) & 0.08 & $(-0.15,0.30)$ & 0.66 & 0.51 \\
\hline 70 Intensity (vs. 50 Intensity) & 0.36 & $(0.13,0.58)$ & 3.09 & $0.002 *$ \\
\hline \multicolumn{5}{|c|}{ Model 2-Outcome: d' /a-i/ for Overall Sample } \\
\hline Intercept & 1.31 & $(0.81,1.80)$ & 5.21 & $<0.001$ \\
\hline Hearing Age at Test & -0.022 & $(-0.07,0.02)$ & -0.97 & 0.33 \\
\hline Mild HL (vs. Normal) & -0.052 & $(-0.38,0.28)$ & -0.31 & 0.76 \\
\hline Mod + HL (vs. Normal) & -0.45 & $(-0.86,-0.04)$ & -2.14 & $0.03 *$ \\
\hline 60 Intensity (vs. 50 Intensity) & 0.149 & $(-0.09,0.39)$ & 1.21 & 0.23 \\
\hline 70 Intensity (vs. 50 Intensity) & 0.184 & $(-0.06,0.43)$ & 1.47 & 0.14 \\
\hline
\end{tabular}

Note. Summary of data using generalized estimating equations (GEE) with an exchangeable working correlation structure to account for the multiple observations across level per individual. * denotes statistical significance.

Table 5. Linear regression models for $\mathrm{CHH}$ cohort only.

\begin{tabular}{|c|c|c|c|c|}
\hline Covariate & Estimate & $95 \% \mathrm{CI}$ & Wald Statistic & $p$ Value \\
\hline \multicolumn{5}{|c|}{ Model 1HL-Outcome: d' /ba-da/ for HL Only } \\
\hline Intercept & -0.43 & $(-1.59,0.73)$ & -0.73 & 0.47 \\
\hline Hearing Age at Test & 0.03 & $(-0.06,0.12)$ & 0.61 & 0.55 \\
\hline Mod+ HL (vs. Mild HL) & 0.15 & $(-0.30,0.61)$ & 0.66 & 0.51 \\
\hline 60 Intensity (vs. 50 Intensity) & 0.09 & $(-0.24,0.43)$ & 0.54 & 0.59 \\
\hline 70 Intensity (vs. 50 Intensity) & 0.47 & $(0.17,0.77)$ & 3.08 & $0.002 *$ \\
\hline Aided SII at $50 \mathrm{~dB}$ & 1.01 & $(-0.17,2.19)$ & 1.67 & 0.09 \\
\hline \multicolumn{5}{|c|}{ Model 2HL-Outcome: d' /a-i / for HL Only } \\
\hline Intercept & 1.15 & $(0.30,1.99)$ & 2.65 & 0.01 \\
\hline Hearing Age at Test & -0.05 & $(-0.11,0.01)$ & -1.61 & 0.11 \\
\hline Mod+ HL (vs. Mild HL) & -0.24 & $(-0.66,0.18)$ & -1.11 & 0.27 \\
\hline 60 Intensity (vs. 50 Intensity) & -0.21 & $(-0.57,0.16)$ & -1.10 & 0.27 \\
\hline 70 Intensity (vs. 50 Intensity) & 0.08 & $(-0.26,0.42)$ & 0.46 & 0.64 \\
\hline Aided SII at $50 \mathrm{~dB}$ & 0.41 & $(-0.63,1.45)$ & 0.77 & 0.44 \\
\hline
\end{tabular}

Note. Regression for $\mathrm{CHH}$ cohort only, using generalized estimating equations (GEE) with an exchangeable working correlation structure to account for the multiple observations across level per individual. ${ }^{*}$ denotes statistical significance.

\subsection{Aided SII}

To examine the potential association of aided SII on speech perception abilities, the regression models were refit and restricted to only the $\mathrm{CHH}$ group. Aided SII at $50 \mathrm{~dB}$ input ranged from $0.21-0.98$. Aided SII is not significantly associated with speech perception 
for /ba-da/ or /a-i/ when adjusting for age, mild versus moderate+ hearing loss, and presentation level ( $p>0.05$ for all).

\section{Discussion}

This study was designed to compare early speech perception abilities for $\mathrm{CHH}$ who met the EHDI guidelines and a group of $\mathrm{CNH}$ using a clinically feasible protocol. We examined whether performance varied as a function of contrast type (vowel and consonant), presentation level, hearing age, and hearing status. Both $\mathrm{CHH}$ and $\mathrm{CNH}$ were able to discriminate each contrast, however more children, regardless of hearing status, were able to reach criterion on the vowel contrast / a-i/ compared to the consonant contrast / ba-da/. $\mathrm{CHH}$ and $\mathrm{CNH}$ had higher scores on the /ba-da/ contrast at $70 \mathrm{dBA}$ compared to $50 \mathrm{dBA}$; the same benefit was observed for the $\mathrm{CHH}$ when analyzed as a separate cohort. We also found that children with moderate+ hearing losses performed significantly poorer than $\mathrm{CNH}$ across multiple intensity levels for /a-i/. However, children with mild hearing loss did not differ in their performance from $\mathrm{CNH}$ for the contrasts tested in this study. Finally, aided SII was not a significant predictor of speech perception performance.

\subsection{Hearing Status}

Children with moderate+ hearing losses performed significantly poorer than $\mathrm{CNH}$ across multiple intensity levels for / $\mathrm{a}-\mathrm{i} /$ when considering hearing age. We were surprised that no differences in speech perception abilities were observed between $\mathrm{CHH}$ and $\mathrm{CNH}$ for /ba-da/ based on our previous research findings. Of note, all CHH benefited from meeting the EHDI benchmarks. Both groups, when considering chronological age, performed similarly, as $74 \%$ of $\mathrm{CHH}$ and $77 \%$ of $\mathrm{CNH}$ were able to successfully discriminate /a-i/ and $56 \%$ of $\mathrm{CHH}$ and $55 \%$ of $\mathrm{CNH}$ were able to discriminate /ba-da/.

Forty-four percent of $\mathrm{CHH}$ and $45 \% \mathrm{CHH}$ did not reach criterion for /ba-da/ at any level. Among $\mathrm{CNH}$ this is poorer than previously reported where $29 \%$ Uhler [37] and $28 \%$ Nozza [34] of 6-8-month-olds did not reach criterion on /ba-da/. Thus, this sample of $\mathrm{CNH}$ performed poorer on /ba-da/ whereas the $\mathrm{CHH}$ performed about the same as reported in Uhler et al. [37]. Overall, performance on /a-i/ was poorer compared to the performance reported in 2017; here $74 \%$ of $\mathrm{CNH}$ and $77 \%$ of $\mathrm{CHH}$ reached criterion at some presentation level. Previously, $85 \%$ and $95 \%$ respectively, of infants could discriminate /a-i/ [37]. It is possible that these differences in performance may be due to natural variability observed, due to an increase in sample size, and/or increasing the number of testers. In Uhler et al. $[37,38]$ testing was done by a single tester whereas data presented in this paper was gathered by five different testers.

When looking at our data for / ba-da/ discrimination, $56 \%$ of $\mathrm{CHH}$ and $55 \%$ of $\mathrm{CNH}$ reached a criterion, which is poorer than performance on /a-i/ discrimination. The finding that vowel discrimination is easier for infants, regardless of hearing status, is also similar to previously reported speech perception abilities found in the $[26,37,38,48,49,61]$. Overall, we have tripled our sample sizes from work reported in 2017 [37]. While the outcomes on speech perception of the vowel contrast remain similar for all infants and performance remains similar among $\mathrm{CHH}$ for /ba-da/, the same was not true for performance among $\mathrm{CNH}$ on the /ba-da/ contrast. Expressly, comparing results from Uhler et al. [37] to our present findings, performance among $\mathrm{CNH}$ declined from nearly $70 \%$ of 21 participants who were able to discriminate /ba-da/ at some presentation level to $56 \%$ among 70 . These results suggest consideration of the duration of hearing aid use may be necessary when examining speech perception. Additionally, clinical use and implementation in a current manner is feasible for the /a-i/ contrast. We would expect $\mathrm{CNH}$ and children with mild hearing loss to perform similarly. If a child can discriminate /ba-da/ the clinical recommendations are relatively straight forward. However, if a child cannot discriminate the /ba-da/ contrast, clinical recommendations are limited, and further exploration is needed to understand these implications. 
We speculate that better performance on /a-i/ than place of articulation contrasts such as /ba-da/ [26,37,48,49,61,62] may be due to the different roles that vowel and consonants play in language acquisition and learning [63] and that language specific vowels emerge before consonants $[43,46]$. These findings warrant further exploration on the perceptual development of vowels and consonants in $\mathrm{CHH}$. We evaluated each contrast at multiple levels to determine if greater presentation levels improved performance, which we observed for /ba-da/. Replicating our previous work in a larger population of CHH who all benefited from meeting EHDI milestones, wore hearing aids, and had mild to severe hearing loss was an important first step towards expanding our work in the development of speech perception. Assessing infant speech perception would not have been possible before newborn hearing screening and the EHDI milestones. Our work supports that $\mathrm{CHH}$ can successfully discriminate these contrasts and that CHT is an efficient way to evaluate this behavior. However, future work in infant speech perception with $\mathrm{CHH}$ should be expanded to include more challenging phoneme contrasts (e.g., /s-sh/) [64], to examine performance in more realistic listening environments such as in background noise [35], and to explore the test-retest reliability in this population [55].

\subsection{Presentation Level}

$\mathrm{CHH}$ and $\mathrm{CNH}$ performed better on the /ba-da/ contrast at 70 compared to $50 \mathrm{dBA}$. The same benefit was observed for the $\mathrm{CHH}$ when analyzed as a separate cohort, while adjusting for age and severity. This difference in overall performance between 50 and $70 \mathrm{dBA}$ agrees with the previous findings from Nozza [34] where infants performed optimally at $70 \mathrm{dBA}$. Nozza also found that 7/10 infants were not performing greater than chance and that testing at $50 \mathrm{dBA}$ may not be reflective of any discrimination ability. These findings support utilization of a greater intensity (i.e., $70 \mathrm{dBA}$ ) level for /ba-da/ and may lead to improved performance on this contrast in a clinical setting. Further, assessing performance variability at different presentation levels could provide valuable information about how amplification algorithms such as noise cancelation, amplitude compression, or frequency compression might affect perceptual development in $\mathrm{CHH}$. Future research to examine such effects could be useful for optimizing hearing aid fittings at different time points that coincide with developmental changes in perception.

\subsection{Aided SII}

As in our previous work, we examined the relationship between speech perception abilities and aided SII, because of the role it plays in hearing aid fittings $[65,66]$. Among $\mathrm{CHH}$, aided SII is commonly used to determine proper amplification fitting based on an individual's hearing loss. Our present results show that aided SII was not a significant predictor of speech perception for either contrast. These findings are partially inconsistent with our previous work [37] when we found that there was a relationship between speech perception abilities of /a-i/ and aided SII, but not / ba-da / and aided SII. However, other previous studies have shown the aided SII may overpredict speech perception abilities in the pediatric population [64-66] and may not be a reliable indicator of predicted of speech perception performance. One explanation for these discrepancies is that aided SII is a measure derived from the expected intelligibility of acoustic speech properties in a typically functioning auditory system. However, the variability in etiologies and severity of hearing loss, coupled with the variability of amplification and treatment inherently lend to atypical acoustic, perceptual, and physiological characteristics that cannot be captured by such derived measures. These findings support inclusion of speech perception testing in addition to assessing aided SII in the clinic.

\subsection{Limitations}

There is a great deal of variability in performance of infant speech perception. We sought to test a large number of $\mathrm{CHH}$ and $\mathrm{CNH}$, which we achieved. However, even when tripling our sample size of $\mathrm{CHH}$, there is an uneven distribution of children with 
each degree of hearing loss ranging from mild to severe. The high variability can be seen from Figure $3 a, b$ which depicts contrast performance across intensity levels and by hearing status. Variability is often a challenge when assessing this population as infants states change during and across test sessions due to factors such as fatigue, hunger, and interest [55]. Advancing infant speech perception work is challenging due to small sample sizes, high attrition rates, data inclusion/exclusion criteria, and high levels of variability. Cristia et al., [55] assessed test-retest reliability across three sites for varying ages (mean age range: 5.89-11.58 months) on 12 independent yet similar experiments each targeting some speech perception skill. In that study, the test-retest period ranged from 0-18 days within the first test date. Their findings revealed only three experiments with positive test-retest correlations, suggesting results from infant speech perception are highly variable. While we did not have multiple sites involved in testing, we did increase the number of clinicians administering the $\mathrm{CHT}$ assessment compared to our previous studies. Cristia et al.'s findings and close examination of our study highlight the need for replication and increased sample sizes in infant speech perception work.

Furthermore, while we do not anticipate that $\mathrm{CNH}$ are unable to discriminate /ba-da/, our study design did not allow us to explore what it means that $44 \%$ of $\mathrm{CNH}$ were unable to reach criterion. However, this is the first work of its kind to report conditioning in a single test session and to report data for all infants who demonstrated successful conditioning to the task regardless of test performance. Future work should include some measure of test-retest reliability across multiple visits. Of note, a subset of our $\mathrm{CNH}$ was previously reported in Uhler et al. [16], for which they completed cortical evoked potential testing and exhibited passive discrimination of our consonant and vowel contrasts. The group tested in this study also lacks generalizability to $\mathrm{CHH}$ who did not meet the JCIH guidelines. Further research in this area could benefit from $\mathrm{CHH}$ that were either diagnosed with hearing loss and/or treated for hearing loss after the recommended EHDI timeline. However, withholding treatment for experimental purposes would not be ethical.

\section{Conclusions}

The majority of $\mathrm{CHH}(74 \%)$ and $\mathrm{CNH}(77 \%)$ were able to discriminate /a-i/ at intensity levels between 50-70 dBA in a single testing session. Reported data included all children who demonstrated successful conditioning regardless of their speech perception scores. However, there was a slight decline for $\mathrm{CHH}$ who were able to discriminate the /a-i/ contrast compared to our previous studies in which $95 \%$ of $\mathrm{CHH}$ could achieve criterion. When comparing discrimination performance of the consonant contrast, these findings are again similar among $\mathrm{CHH}$ such that $56 \%$ of $\mathrm{CHH}$ were able to discriminate /ba-da/ compared to our work published in 2017. The same pattern of performance did not hold true for $\mathrm{CNH}$ on the /ba-da/ contrast. Similar findings with a larger sample size contribute to the generalizability of our results among a population of $\mathrm{CHH}$ who met EHDI benchmarks. $\mathrm{CHH}$ can perform similarly to $\mathrm{CNH}$ in a quiet environment. This work contributes to the speech perception literature among infants with mild to severe permanent hearing loss and demonstrates the feasibility of using this clinically viable protocol.

Author Contributions: All authors contributed to the research and this paper. Conceptualization and methodology were designed by K.M.U.; validation, K.M.U. and K.A.W.; formal analysis was conducted by K.M.U. and A.M.K.; investigation was conducted by K.M.U. and K.A.W.; resources were provided by K.M.U. and P.M.G.; writing-original draft preparation K.M.U. and contributions by K.A.W.; writing - review and editing were completed by all authors; visualization, K.M.U. and P.M.G.; supervision, K.M.U.; project administration, K.M.U.; funding acquisition, K.M.U. and P.M.G. All authors have read and agreed to the published version of the manuscript.

Funding: Funding for this research was provided by the National Institutes of Health-National Institute on Deafness and other Communication Disorders 1K23DC01358; National Organization of Hearing Research (http:/ / www.nohrfoundation.org, accessed on 25 September 2021) and by CCTSI $=$ NIH/NCRR Colorado CTSI Grant Number UL1 TR001082 to author KU. National Institute on Disability, Independent Living, and Rehabilitation Research (NIDILRR \#90RE5020) to author 
PMG, and by the NIDILRR is a Center within the Administration for Community Living (ACL), Department of Health and Human Services (HHS), USA. The contents of this research manuscript do not necessarily represent the policy of NIDILRR, ACL, or HHS, and you should not assume endorsement by the Federal Government.

Institutional Review Board Statement: The study was conducted according to the guidelines of the Declaration of Helsinki, and approved by the Institutional Review Board of University of Colorado (protocol code 13-0342 and 1 April 2020).

Informed Consent Statement: All infants were given written consent by a parent to participate in the study as approved by the Colorado Multiple Institutional Review Board.

Data Availability Statement: De-identified data will be made available after institutional review and approval.

Acknowledgments: We would like to express our gratitude to all families, Colorado Home Intervention Program, and audiologists who participated in this project.

Conflicts of Interest: The authors declare no conflict of interest with regard to the funding source for this study.

\section{References}

1. Joint Committee on Infant Hearing (JCIH). Year 2007 position statement: Principles and guidelines for early hearing detection and intervention programs. Pediatrics 2007, 120, 898-921. [CrossRef]

2. Mayne, A.M.; Yoshinaga-Itano, C.; Sedey, A.L.; Carey, A. Expressive vocabulary development of infants and toddlers who are deaf or hard of hearing. Volta Rev. 1998, 100, 1-28.

3. Vohr, B.; Jodoin-Krauzyk, J.; Tucker, R.; Topol, D.; Johnson, M.J.; Ahlgren, M.; Pierre, L.S. Expressive vocabulary of children with hearing loss in the first 2 years of life: Impact of early intervention. J. Perinatol. 2011, 31, 274-280. [CrossRef]

4. Walker, E.A.; Tomblin, J.B.; Moeller, M.P.; Oleson, J.J.; Harrison, M.; Ambrose, S.E. Language Outcomes in Young Children with Mild to Severe Hearing Loss. Ear Hear. 2015, 36, 76S-91S.

5. Yoshinaga-Itano, C.; Sedey, A.L.; Coulter, D.K.; Mehl, A.L. Language of early and later-identified children with hearing loss. Pediatrics 1998, 102, 1161-1171. [CrossRef]

6. Sininger, Y.S.; Grimes, A.; Christensen, E. Auditory development in early amplified children: Factors influencing auditory-based communication outcomes in children with hearing loss. Ear Hear. 2010, 31, 166-185. [CrossRef]

7. Spivak, L.; Sokol, H.; Auerbach, C.; Gershkovich, S. Newborn hearing screening follow-up: Factors affecting hearing aid fitting by 6 months of age. Am. J. Audiol. 2009, 18, 24-33. [CrossRef]

8. Moeller, M.P. Early intervention and language development in children who are deaf and hard of hearing. Pediatrics 2000, 106, E43. [CrossRef] [PubMed]

9. Halpin, K.S.; Smith, K.Y.; Widen, J.E.; Chertoff, M.E. Effects of universal newborn hearing screening on an early intervention program for children with hearing loss, birth to 3 yr of age. J. Am. Acad. Audiol. 2010, 21, 165-175. [CrossRef] [PubMed]

10. Kennedy, C.R.; McCann, D.C.; Campbell, M.J.; Law, C.M.; Mullee, M.; Petrou, S.; Watkin, P.; Worsfold, S.; Yuen, H.M.; Stevenson, J. Language Ability after Early Detection of Permanent Childhood Hearing Impairment. N. Engl. J. Med. 2006, 354, $2131-2141$. [CrossRef]

11. Yoshinaga-Itano, C.; Sedey, A.L.; Wiggin, M.; Chung, W. Early Hearing Detection and Vocabulary of Children With Hearing Loss. Pediatrics 2017, 140, e20162964. [CrossRef] [PubMed]

12. Pittman, L.; Stewart, E.; Willman, A.; Odgear, I.S. Word Recognition and Learning: Effects of Hearing Loss and Amplification Feature. Trends Hear. 2017, 21, 2331216517709597. [CrossRef] [PubMed]

13. Qi, S.; Mitchell, R.E. Large-scale academic achievement testing of deaf and hard-of-hearing students: Past, present, and future. J. Deaf Stud. Deaf Educ. 2012, 17, 1-18. [CrossRef]

14. Tomblin, J.B.; Harrison, M.; Ambrose, S.E.; Walker, E.A.; Oleson, J.J.; Moeller, M.P. Outcomes of Children Who Are Hard of Hearing. Ear Hear. 2015, 36, 14S-23S. [CrossRef]

15. Walker, E.A.; Sapp, C.; Dallapiazza, M.; Spratford, M.; McCreery, R.W.; Oleson, J.J. Language and reading outcomes in fourthgrade children with mild hearing loss compared to age-matched hearing peers. Lang. Speech Hear. Serv. Sch. 2020, 51, 17-28. [CrossRef]

16. Uhler, K.M.; Hunter, S.K.; Gilley, P.M. Mismatched Response (MMR) predicts behavioral speech discrimination outcomes in infants with hearing loss and normal hearing. Infancy 2021, 26, 327-348. [CrossRef] [PubMed]

17. Uhler, K.; Warner-Czyz, A.; Gifford, R. Pediatric minimum speech test battery. J. Am. Acad. Audiol. 2017, 28, 232-247. [CrossRef]

18. Ching, T.Y.C.; Dillon, H.; Leigh, G.; Cupples, L. Learning from the Longitudinal Outcomes of Children with Hearing Impairment (LOCHI) study: Summary of 5-year findings and implications. Int. J. Audiol. 2017, 57, S105-S111. [CrossRef] [PubMed]

19. Kuhl, P.K.; Conboy, B.T.; Padden, D.; Nelson, T.; Pruitt, J. Early Speech Perception and Later Language Development: Implications for the "Critical Period". Lang. Learn. Dev. 2005, 1, 237-264. [CrossRef] 
20. Ching, T.Y.C.; Dillon, H.; Button, L.; Seeto, M.; van Buynder, P.; Marnane, V.; Cupples, L.; Leigh, G. Age at Intervention for Permanent Hearing Loss and 5-Year Language Outcomes. Pediatrics 2017, 140, e20164274. [CrossRef]

21. Tomblin, J.B.; Oleson, J.J.; Ambrose, S.E.; Walker, E.; Moeller, M.P. The Influence of Hearing Aids on the Speech and Language Development of Children With Hearing Loss. JAMA Otolaryngol. Head Neck Surg. 2014, 104, 403-409. [CrossRef]

22. Uhler, K.; Gifford, R.H. Current trends in pediatric cochlear implant candidate selection and postoperative follow-up. Am. Speech Hear. Assoc. Monogr. 2014, 23, 309-325. [CrossRef] [PubMed]

23. Horn, D.L.; Houston, D.M.; Miyamoto, R.T. Speech discrimination skills in deaf infants before and after cochlear implantation. Audiol. Med. 2007, 5, 232-241. [CrossRef]

24. Miyamoto, R.T.; Houston, D.M.; Kirk, K.I.; Perdew, A.E.; Svirsky, M.A. Language development in deaf infants following cochlear implantation. Acta Otolaryngol. 2003, 123, 241-244. [CrossRef]

25. Phan, J.; Houson, D.; Ruffin, D.; Ting, J.; Holt, R.F. Factors Affecting Speech Discrimination in Children with Cochlear Implants: Evidence from Early Implanted Infants. J. Am. Acad. Audiol. 2016, 27, 480-488. [CrossRef] [PubMed]

26. Uhler, K.; Yoshinaga-Itano, C.; Gabbard, S.A.; Rothpletz, A.M.; Jenkins, H. Longitudinal infant speech perception in young cochlear implant users. J. Am. Acad. Audiol. 2011, 22, 129-142. [CrossRef]

27. Aslin, R.N.; Smith, L.B. Perceptual Development. Annu. Rev. Psychol. 1998, 39, 435-473. [CrossRef]

28. Holt, R.F.; Kirk, K.I.; Hay-McCutcheon, M. Assessing multimodal spoken word-in-sentence recognition in children with normal hearing and children with cochlear implants. J. Speech Lang. Hear. Res. 2011, 54, 632-657. [CrossRef]

29. Newman, R.; Ratner, N.B.; Jusczyk, A.M.; Jusczyk, P.W.; Dow, K.A. Infants' early ability to segment the conversational speech signal predicts later language development: A retrospective analysis. Dev. Psychol. 2006, 42, 643-655. [CrossRef]

30. Tsao, F.M.; Liu, H.M.; Kuhl, P.K. Speech perception in infancy predicts language development in the second year of life: A longitudinal study. Child Dev. 2004, 75, 1067-1084. [CrossRef]

31. Singh, L.; Reznick, J.; Xuehua, L. Infant Word Segmentation and Childhood Vocabulary Development: A Longitudinal Analysis. Dev. Sci. 2012, 15, 482-495. [CrossRef] [PubMed]

32. Moore, J.M.; Thompson, G.; Thompson, M. Auditory localization of infants as a function of reinforcement conditions. J. Speech Lang. Hear. Disord. 1975, 40, 29-34. [CrossRef] [PubMed]

33. Eilers, R.E.; Morse, P.A.; Gavin, W.J.; Oller, D.K. Discrimination of voice onset time in infancy. J. Acoust. Soc. Am. 1981, 70, 955-965. [CrossRef] [PubMed]

34. Nozza, R.J. Infant speech-sound discrimination testing: Effects of stimulus intensity and procedural model on measures of performance. J. Acoust. Soc. Am. 1987, 81, 1928-1939. [CrossRef] [PubMed]

35. Nozza, R.J.; Miller, S.L.; Rossman, R.N.; Bond, L.C. Reliability and validity of infant speech-sound discrimination-in-noise thresholds. J. Speech Hear. Res. 1991, 34, 643-650. [CrossRef] [PubMed]

36. Nozza, R.J. Thresholds are not enough: Understanding how infants' Process. Speech has a role in how we manage hearing loss. In A Sound Foundation through Early Amplification; Phonak AG: Stäfa, Switzerland, 2000; pp. 47-54. Available online: https://www.phonakpro.com/content/dam/phonakpro/gc_hq/en/events/2016/international_pediatric_audiology_ conference_atlanta/chapter_14_moodie_draft.pdf (accessed on 25 September 2021).

37. Uhler, K.M.; Gifford, R.H.; Forster, J.E.; Anderson, M.; Tierney, E.; Claycomb, S.D.; Werner, L.A. Refining Stimulus Parameters in Assessing Infant Speech Perception Using Visual Reinforcement Infant Speech Discrimination in Infants with and without Hearing Loss: Presentation Level. J. Am. Acad. Audiol. 2017, 29, 847-854. [CrossRef]

38. Uhler, K.M.; Baca, R.; Dudas, E.; Fredrickson, T. Refining Stimulus Parameters in Assessing Infant Speech Perception Using Visual Reinforcement Infant Speech Discrimination: Sensation Level. J. Am. Acad. Audiol. 2015, 26, 807-814. [CrossRef]

39. Werker, J.F.; Shi, R.; Desjardins, R.; Pegg, J.E.; Polka, L.; Patterson, M. Three methods for testing infant speech perception. In Perceptual Development: Visual, Auditory, and Speech Perception in Infancy; Slater, A., Ed.; Psychology Press: New York, NY, USA, 1998; pp. 389-420.

40. Eilers, R.E.; Wilson, W.R.; Moore, J.M. Developmental changes in speech discrimination in infants. J. Speech Hear. Res. 1977, 20, 766-780. [CrossRef]

41. Moog, J.; Geers, A. Early Speech Perception Test; Central Institute for the Deaf: St. Louis, MO, USA, 1990.

42. Ertmer, D.J.; Miller, C.M.; Quesenberry, J.K. The Open and Closed Set Test; Purdue University: West Lafayette, IN, USA, 2004.

43. Werker, J.F.; Tees, R.C. Cross-language speech perception: Evidence for perceptual reorganization during the first year of life. Infant Behav. Dev. 1984, 7, 49-63. [CrossRef]

44. Kuhl, P.K.; Williams, K.A.; Lacerda, F.; Stevens, B.; Lindblom, K.N. Linguistic Experience Alters Phonetic Perception in Infants by 6 months of Age. Science 1992, 255, 606-608. [CrossRef]

45. Kuhl, P.K.; Conboy, B.T.; Coffey-Corina, S.; Padden, D.; Rivera-Gaxiola, M.; Nelson, T. Phonetic learning as a pathway to language: New data and native language magnet theory expanded (NLM-e). Philos. Trans. R. Soc. B Biol. Sci. 2008, 363, 979-1000. [CrossRef] [PubMed]

46. Strange, W.; Jenkins, J.J. Role of linguistic experience in the perception of speech. In Perception and Experience; Pick, J.H.D., Pic, H.J., Eds.; Plenum: New York, NY, USA, 1978.

47. Nozza, R.J.; Wilson, W.R. Masked and unmasked pure-tone thresholds of infants and adults: Development of auditory frequency selectivity and sensitivity. J. Speech Hear. Res. 1984, 27, 613-622. [CrossRef] [PubMed] 
48. Martinez, A.; Eisenberg, L.; Boothroyd, A.; Visser-Dumont, L. Assessing speech pattern contrast perception in infants: Early results on VRASPAC. Otol. Neurotol. 2008, 29, 183-188. [CrossRef]

49. Fredrickson, T. Visual Reinforcement Infant Speech Discrimination: Developing a Method of Performance Analysis. Ph.D. Thesis, University of Colorado Boulder, Boulder, CO, USA, 2010.

50. Boothroyd, A. Speech perception tests and hearing impaired children. In Profound Deafness and Speech Communication; Plant, G., Spens, K.E., Eds.; Whurr: London, UK, 1995; pp. 345-371.

51. Stika, C.J.; Eisenberg, L.S.; Johnson, K.C.; Henning, S.C.; Colson, B.G.; Ganguly, D.H.; DesJardin, J.L. Developmental outcomes of early identified children who are hard of hearing at 12 to 18 months of age. Early Hum. Dev. 2015, 91, 47-55. [CrossRef]

52. Schauwers, K.; Gillis, S.; Daemers, K.; de Beukelaer, C.; Govaerts, P.J. Cochlear Implantation Between 5 and 20 months of Age: The Onset of Babbling and the Audiologic Outcome. Otol. Neurotol. 2004, 25, 263-270. [CrossRef]

53. Govaerts, P.J.; Daemers, K.; Yperman, M.; de Beukelaer, C.; de Saegher, G.; de Ceulaer, G. Auditory speech sounds evaluation $\left(\mathrm{A} \S \mathrm{E}^{\circledR}\right)$ : A new test to assess detection, discrimination, and identification in hearing impairment. Cochlear Implants Int. 2006, 7 , 97-106. [CrossRef]

54. Houston, D.M.; Pisoni, D.B.; Kirk, K.I.; Ying, E.A.; Miyamoto, R.T. Speech perception skills of deaf infants following cochlear implantation: A first report. Int. J. Pediatr. Otorhinolaryngol. 2003, 67, 479-495. [CrossRef]

55. Cristia, A.; Seidl, A.; Singh, L.; Houston, D. Test-Retest Reliability in Infant Speech Perception Tasks. Infancy 2016, 21, 648-667. [CrossRef]

56. American Academy of Audiology (AAA) Task Force on Pediatric Amplification. American Academy of Audiology Clinical Practice Guidelines Pediatric Amplification American Academy of Audiology Clinical Practice Guidelines; American Academy of Audiology: Reston, VA, USA, 2011.

57. Eisenberg, L.S.; Martinez, A.S.; Boothroyd, A. Assessing auditory capabilities in young children. Int. J. Pediatr. Otorhinolaryngol. 2007, 71, 1339-1350. [CrossRef] [PubMed]

58. Macmillan, N.; Creelman, C. Detection Theory: A User's Guide; Lawrence Erlbaum Associates: Mahwah, NJ, USA, 2005.

59. McArdle, R.; Hnath-Chisolm, T. Speech Audiometry. In Handbook of Clinical Audiology, 6th ed.; Katz, J., Medwetsky, L., Burkard, R., Hood, L., Eds.; Lippincott Williams and Wilkins: Baltimore, MD, USA, 2009.

60. McCreery, R.W.; Walker, E.A.; Spratford, M.; Bentler, R.; Holte, L.; Roush, P.; Oleson, J.; van Buren, J.; Moeller, M.P. Longitidinal predictors of aided speech audibility in infants and children. Ear Hear. 2015, 36, 24S-37S. [CrossRef] [PubMed]

61. Boothroyd, A. Auditory Perception of Speech Contrasts by Subjects with Sensorineural Hearing Loss. J. Speech Hear. Res. 1984, 27, 134-144. [CrossRef]

62. van Wieringen, A.; Wouters, J. Natural vowel and consonant recognition by Laura Cochlear implantees. Ear Hear. 1999, 20, 89-103. [CrossRef] [PubMed]

63. Toro, J.M.; Nespor, M.; Mehler, J.; Bonatti, L.L. Finding words and rules in a speech stream: Functional differences between vowels and consonants: Research article. Psychol. Sci. 2008, 19, 137-144. [CrossRef] [PubMed]

64. Scollie, S.; Glista, D.; Seto, J.; Dunn, A.; Schuett, B.; Hawkins, M.; Pourmand, N.; Parsa, V. Fitting Frequency-Lowering Signal Processing Applying the American Academy of Audiology Pediatric Amplification Guideline: Updates and Protocols. J. Am. Acad. Audiol. 2016, 27, 219-236. [CrossRef] [PubMed]

65. Scollie, S. Children's speech Frecognition scores: The speech intelligibility index and proficiency factors for age and hearing level. Ear Hear. 2008, 29, 543-556. [CrossRef] [PubMed]

66. McCreery, R.W.; Stelmachowicz, P.G. Audibility-based predictions of speech recognition for children and adults with normal hearing. J. Acoust. Soc. Am. 2011, 130, 4070-4081. [CrossRef] [PubMed] 